\title{
Quantum Optics with Atomic Ensembles and Single Atoms in Cavities
}

\author{
T. E. Northup and H. J. Kimble \\ Norman Bridge Laboratory of Physics 12-33, \\ California Institute of Technology, Pasadena CA 91125
}

\begin{abstract}
Current experiments in our group explore the quantum interface between matter and light, with the goal of achieving coherent control for implementing quantum information protocols and quantum networks. We outline recent progress in this direction, including localization to the ground state of motion for an atom trapped in an optical cavity, observation of strong coupling between single Cesium atoms and a monolithic resonator, and generation and characterization of entanglement stored in remote atomic ensembles.
\end{abstract}

PACS: $42.50 . \mathrm{Pq}, 32.80 .-\mathrm{t}, 42.50 . \mathrm{Ct}, 42.50 . \mathrm{Dv}$

Last year's Nobel Prize in Physics brought attention to the remarkable capabilities that have emerged in recent years in Quantum Optics. The work of Professors J. L. Hall and T. W. Hänsch has provided the community with stable optical frequencies that even "amateurs" can gainfully employ as the basis for future "quantum-state synthesizers" to generate arbitrary states in diverse Hilbert spaces. To understand the zoology of these states, Professor R. J. Glauber has provided a powerful theory of quantum theory for measuring and identifying states that are fundamentally non-classical, and hence of interest in Quantum Information Science.

In addition to achieving control for the generation of quantum states of light, current research in Quantum Optics also strives to implement quantum interfaces between matter and light, that is, to map "arbitrary" quantum states of material systems to photons and back again. To implement such mappings successfully, the underlying physical processes must be robust in the face of diverse imperfections, including most importantly, couplings to various unobserved dissipative mechanisms. All laboratory systems are open quantum systems, so that the effects of decoherence can be minimized but never completely avoided. Hence, operational protocols must incorporate the capability both to detect and "fix" inevitable mistakes arising from the dynamics of real physical systems.

Within this general context, in this article we briefly describe two approaches to coupling quantized light and matter that are currently underway in the Quantum Optics Group at Caltech. The first employs strong coupling within the setting of cavity quantum electrodynamics (cavity QED) using a traditional Fabry-Perot cavity [1] and, more recently, a microtoroidal resonator [2]. The second approach creates and characterizes entanglement by writing and reading single excitations to and from a pair of atomic ensembles [3]. 


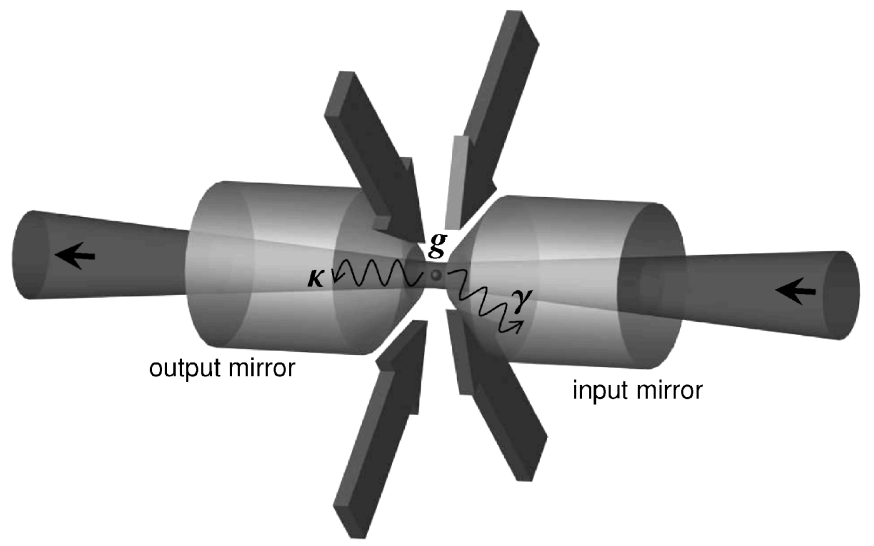

FIGURE 1. A Fabry-Perot cavity with a single atom trapped in a FORT along the cavity axis. Axial beams are also used to probe the atom-cavity system near resonance and to drive Raman transitions between the atomic hyperfine ground states. Lattice beams from the side of the cavity load the atom into the FORT. Atom-cavity coupling $g$ and decoherence rates $\kappa, \gamma$ are illustrated.

\section{CAVITY QED IN THE STRONG COUPLING REGIME}

Current research activities in cavity QED were summarized at ICAP 2006 by Professor Gerhard Rempe. For a more extensive overview of the field, we refer you to his article in these proceedings, as well as to a recent review from our group [4]. The idealized model of cavity QED consists of a single atom localized within a nearly-perfect optical resonator in a regime of strong coupling, for which the rate of coherent atom-cavity coupling $g$ dominates all dissipative mechanisms, including spontaneous emission by the atom (at rate $\gamma_{\perp}$ ) and transmission, scattering, and absorption by the cavity mirrors (at rate $\kappa$ ). In a cavity-mediated setting, one can achieve nonlinear interactions between individual atoms and single photons. Note that this model relies on a method of trapping the atom that decouples its internal and external degrees of freedom.

Our experimental apparatus [5, 6] attempts to realize these conditions. A Fabry-Perot cavity, $42.2 \mu \mathrm{m}$ in length and with a finesse of $4.3 \times 10^{5}$, can be tuned into resonance with the $D_{2}$ line of atomic Cesium at $852 \mathrm{~nm}$. The resulting coherent coupling rate $g=2 \pi \times 34 \mathrm{MHz}$ for the $6 S_{1 / 2}, F=4, m_{F}=4 \leftrightarrow 6 P_{3 / 2}, F=5, m_{F}=5$ transition, so that $g \gg\left(\gamma_{\perp}, \kappa\right)=2 \pi(2.6,4.1) \mathrm{MHz}$. The system thus has critical photon number $n_{0}=\gamma_{\perp}^{2} /\left(2 g^{2}\right)=2.9 \times 10^{-3}$ and critical atom number $N_{0}=2 \kappa \gamma_{\perp}^{2} / g^{2}=1.8 \times 10^{-2}$. Laser-cooled atoms are loaded into a standing-wave far-off-resonance trap (FORT) along the cavity axis. Within the FORT, atoms have a trap lifetime of $\sim 3 \mathrm{~s}$, axial and radial motion $(\Delta z, \Delta \rho) \sim(33 \mathrm{~nm}, 5.5 \mu \mathrm{m})$, and "temperature" $<100 \mu \mathrm{K}$. By exploiting Cesium's "magic" wavelength at $\sim 935 \mathrm{~nm}$ for the FORT beam, we create an "ion trap" for neutral atoms whereby transformations via excited states have minimal decoherence $[5,7,8,9]$.

Following our demonstration of $3.1 \pm 0.4 \mathrm{~s}$ trap lifetimes in 2003 [6], we have taken a series of important steps toward full control of our cavity QED system. We have used 
a sequence of classical pulses to generate single photons on demand, a critical resource for quantum information science [10]. By monitoring the transmission of a probe laser along the cavity axis, we have observed a "digital" readout of the intracavity atom number, allowing us to distinguish in real time between $N=0,1,2 \ldots$ trapped atoms [11]. Measurement of the vacuum-Rabi spectrum for one and the same atom established the capability to carry out a complex quantum "protocol" with one trapped atom, acquiring information that previously required $N \sim 10^{3}-10^{5}$ atoms to obtain [12].

Accessing the strong coupling regime $g_{0} \gg\left(\gamma_{\perp}, \kappa\right)$ enables us to move beyond traditional nonlinear optics, as in our observation of "photon blockade" [13]. Our point of departure in this experiment is the solid-state phenomenon of Coulomb blockade, whereby electron transport through a small metallic island is profoundly altered if the charging energy of the island $e^{2} / 2 C \gg k_{B} T$, the thermal energy [14, 15]. Following Refs. $[16,17]$, we have investigated an analogous effect for photon transport through an optical cavity containing a single atom. Here the anharmonicity of the Jaynes-Cummings ladder of eigenstates provides the blockade mechanism: absorption of a first photon on resonance with the lower dressed state of our system prevents the absorption of a second photon, thereby leading to a sub-Poissonian and antibunched photon stream for the field transmitted by the atom-cavity system. This phenomenon requires strong coupling, since incoherent decay mechanisms act to broaden the transmission spectrum and destroy the blockade effect.

\section{Localization to the ground state of motion}

Recently we have turned our attention to controlling atomic motion within our cavity via the technique of Raman transitions. In an earlier version of this project, we made use of the dipole-trap standing wave at $935.6 \mathrm{~nm}$ as one arm of a Raman pair [4]. More recently, we have implemented a new scheme by driving an adjacent cavity mode at $945.6 \mathrm{~nm}$ with two phase-locked lasers separated in frequency by the Cesium groundstate hyperfine splitting plus an additional Raman detuning $\delta_{R}$. We then scan $\delta_{R}$ in order to drive transitions between vibrational states of the $F=3$ to $F=4$ levels. Specifically, if $\omega_{\text {axial }} \approx 2 \pi \times 530 \mathrm{MHz}$ is the axial frequency at the bottom of our trapping potential, then by tuning $\delta_{R}$ to $-\omega_{\text {axial }}$ we can make use of the red Raman sideband to remove a quantum of vibrational energy from the system each time we drive the $F=3 \rightarrow 4$ transition. Note that the axial Lamb-Dicke parameter for our system $\eta_{\text {axial }} \approx 0.057 \ll 1$, a prerequisite for efficient cooling [18]. We first incorporated this technique in Ref. [12] as a mechanism for axial cooling between intervals of probing the atom-cavity system.

Pioneering work with trapped ions [19] and atoms in optical lattices [20, 21, 22] has demonstrated cooling to the vibrational ground state of motion via resolved Raman sideband cooling, thereby enabling quantum control of center-of-mass motion. Following this lead, we have measured the distribution of vibrational excitation which results from sideband cooling of a single atom trapped in the cavity mode. These measurements require a method for single-atom quantum-state detection, much as is the case for single trapped ions [18]. To solve this problem, we have adapted quantum-jump spectroscopy to the context of cavity QED, as describe in more detail in Ref. [1]. Overall, our scheme 

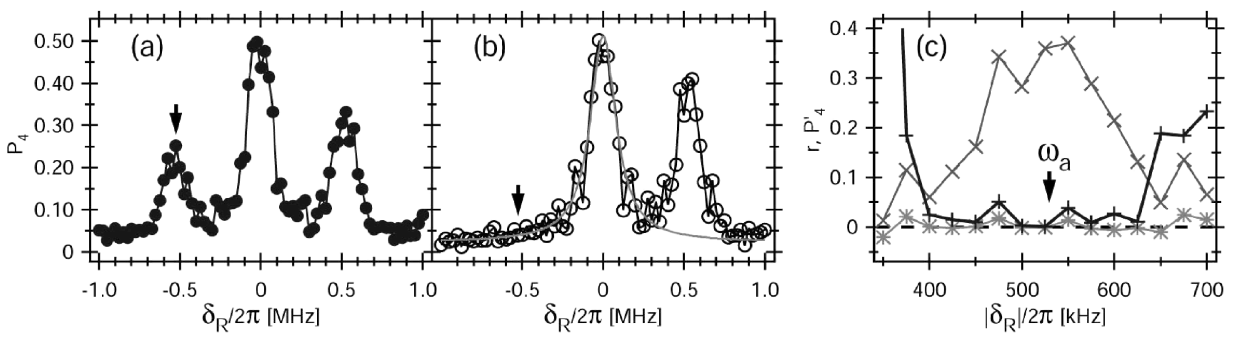

FIGURE 2. Raman spectrum of an intracavity atom, with (a) $250 \mu \mathrm{s}$ and (b) $5 \mathrm{~ms}$ of sideband cooling, the latter with a Lorentzian fitted to the carrier. (c) $\operatorname{Red}(*)$ and blue $(\times)$ motional sidebands (same data as in panel b), as well as their ratio (+), after subtracting the Lorentzian contribution of the carrier. The suppression of the red sideband indicates cooling to motional ground state with probability $P_{0} \approx 0.95 \pm 0.05[1]$.

allows us to distinguish between the possibilities of (1) the atom in $F=4$ and (2) the atom in $F=3$ or no atom with a confidence level $>98 \%$ within $100 \mu$ s.

With the capability for atomic state readout in hand, we perform measurements as follows: a variable duration of sideband cooling is applied to an intracavity atom (with $\left.\delta_{R} \simeq-\omega_{\text {axial }}\right)$; the atom is then optically pumped to the $F=3$ ground state; a Raman pulse with arbitrary detuning $\delta_{R}$ is applied; and finally, the probability $P_{4}$ that this pulse has transferred the atom to the state $F=4$ is measured via our cavity-QED version of quantum-jump spectroscopy.

In Figure 2, we plot $P_{4}$ as a function of Raman detuning $\delta_{R}$. In (a), the atom has received only $250 \mu \mathrm{s}$ of cooling with $\delta_{R} \simeq-\omega_{\text {axial }}$, with then clearly resolved red and blue motional sidebands observed at $\delta_{R} / 2 \pi \sim \pm \omega_{\text {axial }} / 2 \pi= \pm 530 \mathrm{kHz}$. Note that the cooling has already induced an asymmetry in sideband heights. In (b), after $5 \mathrm{~ms}$ of cooling, the red sideband is now so strongly suppressed that it is indistinguishable from a Lorentzian fit to the carrier. We find that the ratio of the red to blue sideband amplitudes $r \approx 0.05$, implying a probability $P_{0} \approx 0.95 \pm 0.05$ to occupy the $n=0$ state. This corresponds to axial localization of the atom within $\simeq 8 \mathrm{~nm}$. Significantly, both simulation and measurement indicate that this is a robust scheme, insensitive to exact experimental parameters such as $\delta_{R}$ and the intensity of the repumping field [1].

Our measurements represent the first observation of cooling to the ground state for a strongly coupled atom-cavity system. We thereby enter the quantum regime in cavity QED not only for the internal degrees of freedom of our system (i.e., the atomic dipole and cavity field), but also for the external degrees of freedom (i.e., the center-of-mass motion of the atom). This new capability opens the door for future quantum measurement of atomic position beyond the standard quantum limit [23], as well as for quantum state exchange between motion and light [24]. 


\section{Strong coupling with a monolithic resonator}

The recent results described above are the product of a decades-long effort in our group to achieve and exploit strong coupling in the optical domain by utilizing optical resonators formed by two spherical mirrors in a Fabry-Perot configuration. These techniques have carried us from the realm of traditional optical physics into a regime of nonlinear interactions with single atoms and photons. However, we must recognize that there are inherent limitations to this approach, due to the serious technical challenges involved in further improvement of mirror coatings and in scaling to large numbers of Fabry-Perot resonators. For example, the realization of large-scale quantum networks requires the capability to interconnect many "quantum nodes." This task is better-suited to an implementation such as an array of microresonator nodes on a chip, each containing individual trapped atoms. The "quantum channels" to connect these nodes would then be optical fiber, with strong interactions in cavity QED providing an efficient interface between light and matter.

Toward this end, we have recently achieved strong coupling between individual Cesium atoms and the whispering gallery modes of a fused silica microtoroidal resonator [2], which represents the first demonstration of single-atom strong coupling in the optical regime outside of Fabry-Perot systems. Our microcavities have been developed by the group of Professor K. Vahala at Caltech. They are lithographically fabricated as arrays of $\mathrm{SiO}_{2}$ microdisks on a silicon microchip, which are then illuminated by a $\mathrm{CO}_{2}$ laser to form a smooth toroidal resonator [25]. Quality factors $Q \simeq 4 \times 10^{8}$ have been demonstrated at telecommunications wavelengths, with projected values of $Q \sim 7 \times 10^{9}$ for operation at $850 \mathrm{~nm}$. This would correspond to $g_{0} / 2 \pi \sim 400 \mathrm{MHz}$, a critical atom number $N_{0} \sim 5 \times 10^{-7}$, and a critical photon number $n_{0} \sim 3 \times 10^{-5}$ [26], surpassing the best achievements to date in Fabry-Perot cavities. In our initial experiment, we infer a more modest value $g_{0}^{m} / 2 \pi=50 \pm 12 \mathrm{MHz}$, which nevertheless places us firmly in the strong coupling regime [2].

As illustrated in Figure 3, we position a chip containing 35 microtoroids within our vacuum chamber so as to couple a tapered fiber to one of the toroids. Specifically, we achieve critical coupling of the resonator and fiber. Laser-cooled Cesium atoms are then collected and dropped from above the microtoroid, with some small number of atoms transiting through the external evanescent field of the resonator. The interaction of one atom with the evanescent field destroys the condition of critical coupling, leading to an increase in the forward propagating power $P_{F}$ in the fiber taper.

We record single-atom transits on photon counters at the fiber output, with a typical detection trace pictured in Figure 4(a), while for comparison, (b) displays background counts in the absence of atoms. The sharp peaks correspond to individual atom transits and have duration $\Delta t \approx 2 \mu s$. The temporal profile of these events shows good agreement with our calculation based on the field distribution in Figure 3(b).

To infer the maximum accessible coupling rate, we record the dependence of $P_{F}$ on the frequency detuning between the atom and cavity resonances $\Delta_{A C}$ for individual atom transits, and compare these measurements with a new theoretical model which we have developed. Details of this model can be found in the Supplemental Information that accompanies Ref. [2]. We find $g_{0}^{m} / 2 \pi=50 \pm 12 \mathrm{MHz}$, thereby achieving the conditions 


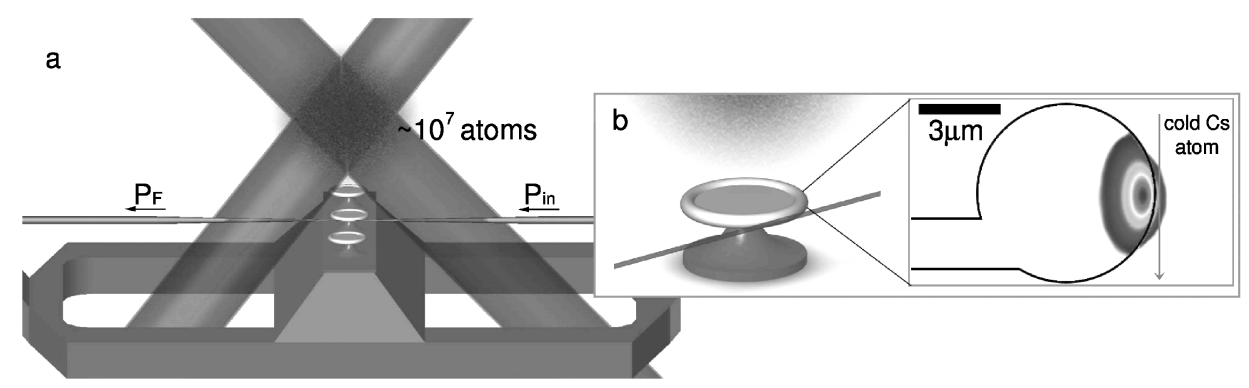

FIGURE 3. (a) Schematic of our experiment showing a cloud of cold Cs and associated trapping lasers above an array of microtoroidal resonators. Light from the probe beam $P_{\text {in }}$ is coupled into a resonator by way of the fiber taper, with the forward propagating output $P_{F}$ coupled into the taper. (b) Illustration of an $\mathrm{SiO}_{2}$ microtoroidal resonator, fiber taper, and atom cloud above. The calculated field distribution for the lowest order resonator mode is shown by the color contour plot on the right. Cold Cs atoms fall through the external evanescent field of this mode and are thereby strongly coupled to the resonator's field [2].
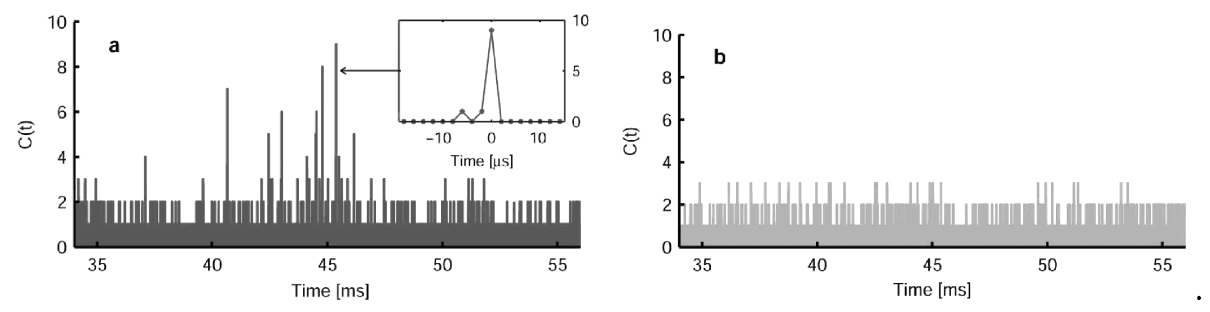

FIGURE 4. (a) Single-photon counting events $C(t)$ from the field $P_{F}$ as a function of time $t$ after the release of a cloud of cold atoms recorded for time bins of $\delta t=2 \mu$ s duration. (a) $C(t)$ for the case with atoms dropped and (b) $C(t)$ for the reference with no atom cloud. The inset in (a) shows the time profile for a single atom transit [2]

for strong coupling, namely $g_{0}^{m}>\left(\gamma_{\perp}, \kappa, \Delta t^{-1}\right)$, where the rate of atomic spontaneous decay $\gamma_{\perp} / 2 \pi=2.6 \mathrm{MHz}$, the cavity field decay rate $\kappa / 2 \pi=18 \pm 3 \mathrm{MHz}$, and $\Delta t \approx 2 \mu \mathrm{s}$ is the average transit duration through the evanescent field of the toroidal resonator.

A significant feature of the microtoroidal resonators is the potential for nearly lossless input-output coupling via tapered fiber, with ideality measured in excess of $99.97 \%$ [27]. Also, we are encouraged by the potential for continued improvement of the resonator quality factor; for example, we have previously measured $Q \approx 8 \times 10^{9}$ for a silica microsphere, with $Q_{\text {projected }} \geq 10^{10}$ [28]. Taken together, these findings indicate that microtoroidal resonators provide a realistic pathway to scalable quantum networks, offering both strong coupling and high intrinsic efficiency for input/output operations.

\section{ENTANGLEMENT BETWEEN REMOTE ATOMIC ENSEMBLES}

While distributed quantum entanglement between cavity QED systems remains a goal, we have recently demonstrated heralded entanglement between remote atomic ensem- 

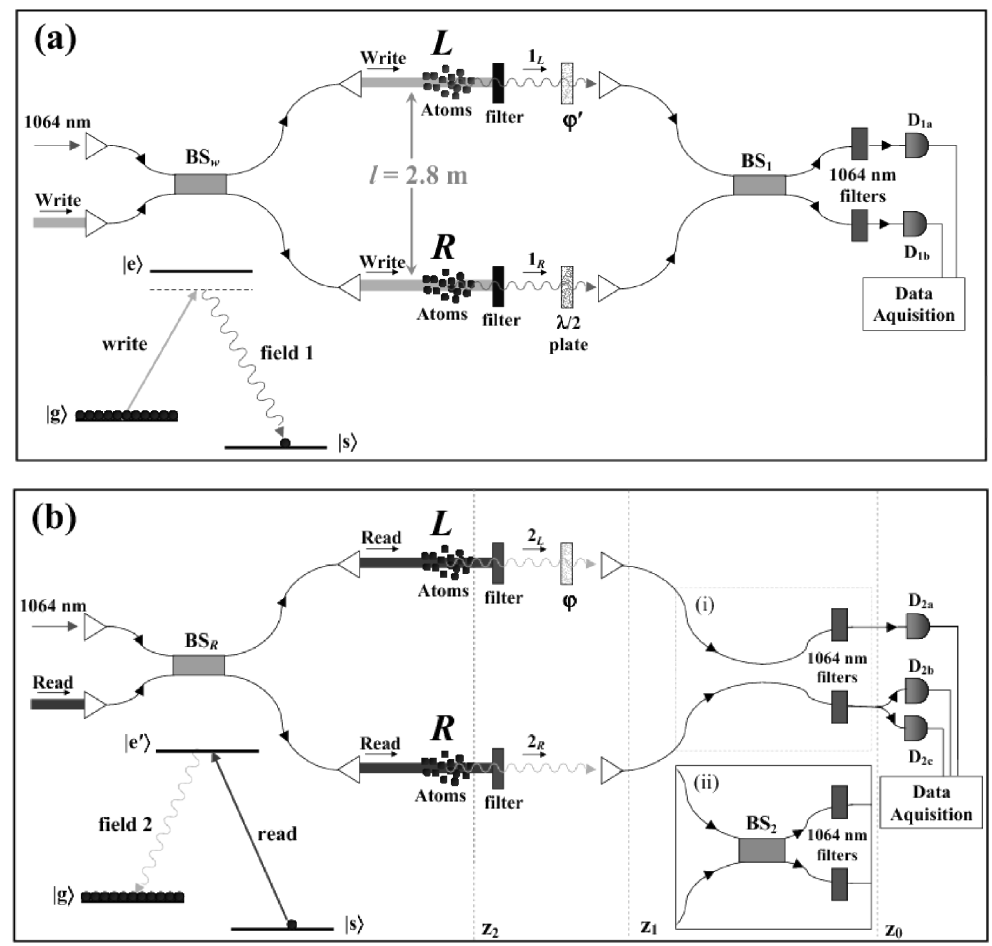

FIGURE 5. Schematic of the entanglement experiment [3] between atomic ensembles, where entanglement preparation is depicted in (a) and detection in (b). Cesium atoms are cooled and trapped in two independent magneto-optical traps at locations $L$ and $R$ separated by $2.8 \mathrm{~m}$.

bles [3] based upon the proposal by Duan, Lukin, Cirac and Zoller (DLCZ) [29]. Among the salient features of this remarkable protocol are its built-in capability for entanglement purification, its use of collectively enhanced coupling, its application as a quantum memory, and finally, its resilience to realistic noise sources.

To entangle two atomic ensembles via the DLCZ protocol, we first apply a weak "write" pulse to both ensembles, prepared initially with all atoms in a ground state $|a\rangle$ (Fig. 5a) [30]. The write pulse connects $|a\rangle$ with the excited state $|e\rangle$, from which an atom can decay to a second ground state $|b\rangle$, thereby emitting a photon (field 1). In the ideal case, if the write pulse is sufficiently weak, the detection of this photon in the forwardscattered direction projects the ensemble onto a state with a single symmetric collective spin excitation. Now, if the fields emitted by both ensembles are combined on a 50/50 beamsplitter with photon counters at both outputs, and if we cannot know in principle from which ensemble the photon was emitted, then in the ideal case, conditioned upon a detection event at $\left(D_{1 a}, D_{1 b}\right)$, an entangled state is created and stored in the two $(L, R)$ ensembles:

$$
\left|\psi_{L, R}\right\rangle=\left|0_{L}\right\rangle\left|1_{R}\right\rangle \pm e^{i \phi}\left|0_{R}\right\rangle\left|1_{L}\right\rangle
$$


The operational verification of entanglement, however, is a more difficult task, which is accomplished by mapping the joint state of the ensembles onto optical fields (Fig. 5 b). After a delay, we apply a strong "read" pulse on the $|b\rangle \rightarrow|e\rangle$ transition to both ensembles, thereby reliably mapping the matter state of our two ensembles onto a field state for two beams of light (field 2) [30]. By measuring the quantum correlation of the fields, we perform quantum-state tomography to determine the density matrix $\rho_{2_{L}, 2_{R}}$ for the fields $2_{L}, 2_{R}$. The resulting density matrix components allow us to calculate the concurrence $C$ [31], where $C>0$ demonstrates unambiguously a non-zero degree of entanglement between the optical fields. At the detectors for field 2 shown in Figure 5, we find $C_{L, R}^{1 a} \geq(2.4 \pm 0.6) \times 10^{-3}>0$ and $C_{L, R}^{1 b} \geq(1.9 \pm 0.6) \times 10^{-3}>0$, depending upon which of the detectors $D_{1 a}, D_{1 b}$ registered the projection event for field 1 . We thereby establish entanglement between two atomic ensembles spatially separated by about 3 meters, which jointly share one quantum of excitation [3].

Beyond these observations, more recently we have made the first direct measurement of decoherence in an atom-light entanglement experiment [32]. Here we generate entanglement between a collective atomic excitation and the polarization of a single photon. After a storage time $\tau$, we map the atomic state onto a second field and measure the polarization correlation of this field with that of the first field. Entanglement is characterized by the violation of a Bell inequality, which we observe to persist for storage times up to $21 \mu \mathrm{s}, 700$ times longer than the duration of the original excitation pulses.

Both atomic ensembles and cavity QED systems are excellent candidates for nodes within quantum networks, at which novel quantum states could be generated, processed, and stored. A principal motivation for our endeavor to control the quantum light-matter interface is the need to link the stationary quantum bits in these nodes together via quantum channels, which would transport and distribute entanglement as "flying qubits" ( $a k a$, photons). While our current work represents only the first steps in this direction, the long-term effort to construct such quantum networks offers both exciting technical challenges and the opportunity to explore diverse fundamental scientific questions.

\section{ACKNOWLEDGMENTS}

We gratefully acknowledge coauthors T. Aoki, A. Boca, A. D. Boozer, W. P. Bowen, C. W. Chou, B. Dayan, D. Felinto, J. Laurat, T. J. Kippenberg, R. Miller, A. S. Parkins, S. V. Polyakov, H. de Riedmatten, E. Schomburg, K. J. Vahala, S. J. van Enk, and E. Wilcut. This work was supported by the National Science Foundation, the Army Research Office, and the Disruptive Technology Office of the Department of National Intelligence.

\section{REFERENCES}

1. A. D. Boozer, A. Boca, R. Miller, T. E. Northup, and H. J. Kimble, Phys. Rev. Lett. 97, 083602 (2006).

2. T. Aoki, B. Dayan, E. Wilcut, W. P. Bowen, A. S. Parkins, H. J. Kimble, T. J. Kippenberg, and K. J. Vahala, Nature (2006), to be published. 
3. C. W. Chou, H. de Riedmatten, D. Felinto, S. V. Polyakov, S. J. van Enk, and H. J. Kimble, Nature 438, 828 (2005).

4. R. Miller, T. E. Northup, K. M. Birnbaum, A. Boca, A. D. Boozer, and H. J. Kimble, J. Phys. B: At. Mol. Opt. Phys. 38, S551 (2005).

5. J. Ye, D. W. Vernooy, and H. J. Kimble, Phys. Rev. Lett. 83, 4987 (1999).

6. J. McKeever, J. R. Buck, A. D. Boozer, A. Kuzmich, H.-C. Nägerl, D. M. Stamper-Kurn, and H. J. Kimble, Phys. Rev. Lett. 90 (2003).

7. H. J. Kimble, C. J. Hood, T. W. Lynn, H. Mabuchi, D. W. Vernooy, and J. Ye, “The quantum internet," in Laser Spectroscopy: XIV International Conference, edited by R. Blatt, J. Eschner, D. Leibfried, and F. Schmidt-Kaler, World Scientific, Singapore, 1999, p. 80.

8. H. Katori, T. Ido, and M. Kuwata-Gonokami, J. Phys. Soc. Jpn. 68, 2479 (1999).

9. T. Ido, Y. Isoya, and H. Katori, Phys. Rev. A 61, 061403 (2000).

10. J. McKeever, A. Boca, A. D. Boozer, R. Miller, J. R. Buck, A. Kuzmich, and H. J. Kimble, Science 303, 1992 (2004).

11. J. McKeever, J. R. Buck, A. D. Boozer, and H. J. Kimble, Phys. Rev. Lett. 93, 143601 (2004).

12. A. Boca, R. Miller, K. M. Birnbaum, A. D. Boozer, J. McKeever, and H. J. Kimble, Phys. Rev. Lett. 93, 233603 (2004).

13. K. M. Birnbaum, A. Boca, R. Miller, A. D. Boozer, T. E. Northup, and H. J. Kimble, Nature 436, 87 (2005).

14. T. A. Fulton, and G. J. Dolan, Phys. Rev. Lett. 59, 109 (1987).

15. M. A. Kastner, Rev. Mod. Phys 64, 849 (1992).

16. L. Tian, and H. J. Carmichael, Phys. Rev. A 46, R6801 (1992).

17. A. Immamōglu, H. Schmidt, G. Woods, and M. Deutsch, Phys. Rev. Lett. 79, 1467 (1997).

18. D. Leibfried, R. Blatt, C. Monroe, and D. Wineland, Rev. Mod. Phys. 75 (2003).

19. C. Monroe, D. M. Meekhof, B. E. King, S. R. Jefferts, W. M. Itano, D. J. Wineland, and P. Gould, Phys. Rev. Lett. 75, 4011 (1995).

20. S. E. Hamann, D. L. Haycock, G. Klose, P. H. Pax, I. H. Deutsch, and P. S. Jessen, Phys. Rev. Lett. 80, 4149 (1998).

21. H. Perrin, A. Kuhn, I. Bouchoule, and C. Salomon, Europhys. Lett. 42, 395 (1998).

22. V. Vuletić, C. Chin, A. J. Kerman, and S. Chu, Phys. Rev. Lett. 81, 5768 (1998).

23. P. Storey, M. Collett, and D. Walls, Phys. Rev. Lett. 68, 472 (1992).

24. A. S. Parkins, and H. J. Kimble, J. Opt. B: Quantum Semiclass. Opt. 1, 496 (1999).

25. D. K. Armani, T. J. Kippenberg, S. M. Spillane, and K. J. Valhala, Nature 421, 925 (2003).

26. S. M. Spillane, T. J. Kippenberg, K. J. Vahala, K. W. Goh, E. Wilcut, and H. J. Kimble, Phys. Rev. A 71, 013817 (2005).

27. S. M. Spillane, T. J. Kippenberg, O. J. Painter, and K. J. Valhala, Phys. Rev. Lett. 91, 043902 (2003).

28. D. W. Vernooy, V. S. Ilchenko, H. Mabuchi, E. W. Streed, and H. J. Kimble, Opt. Lett. 23, 247 (1998).

29. L.-M. Duan, M. D. Lukin, J. I. Cirac, and P. Zoller, Nature 414, 413 (2001).

30. J. Laurat, H. de Riedmatten, D. Felinto, C.-W. Chou, E. W. Schomburg, and H. J. Kimble, Optics Express 14, 6912 (2006).

31. W. K. Wootters, Phys. Rev. Lett. 80, 2245 (1998).

32. H. de Riedmatten, J. Laurat, C. W. Chou, E. W. Schomburg, D. Felinto, and H. J. Kimble, Phys. Rev. Lett. (2006), to be published. 